\title{
Avaliação da qualidade de vida em indivíduos hospitalizados portadores de Doença Pulmonar Obstrutiva Crônica
}

\author{
Evaluation of quality of life in \\ hospitalized individuals with chronic \\ obstructive pulmonary disease
}

FisiSenectus . Unochapecó Ano 7, n. 1 - Jan/Jun. 2019 p. $15-22$

Camila Tres Dal Puppo. camiladalpuppo@hotmail.com.br

Fisioterapeuta. Graduada pela Universidade de Passo Fundo (UPF). Atua na Clínica de Pilates, Constantina/RS.

Felipe Hübner. felipe_hubner@hotmail.com

Fisioterapeuta. Graduado pela Universidade de Passo Fundo (UPF). Atua na Clínica Reabilitare, Passo Fundo/RS.

Débora D`Agostini Jorge Lisboa. debydj@yahoo.com.br

Fisioterapeuta. Mestre em Envelhecimento Humano pela Universidade de Passo Fundo (UPF). Fisioterapeuta e coordenadora do Serviço de Fisioterapia do Hospital de Clínicas de Passo Fundo (HCPF). Docente do Curso de Fisioterapia da UPF-RS. Doutoranda em Envelhecimento Humano pela UPF-RS.

\section{Resumo}

Introdução: a Doença Pulmonar Obstrutiva Crônica (DPOC) tem como característica o comprometimento do fluxo aéreo na árvore brônquica, desenvolvendo sintomas variáveis, como expectoração, dispneia e tosse. Tais sintomas da DPOC comprometem a funcionalidade e a vida social dos indivíduos, limitando suas atividades da vida diária podendo assim afetar sua qualidade de vida. Objetivo: o presente estudo teve como objetivo avaliar a qualidade de vida em indivíduos hospitalizados com diagnóstico médico de DPOC por meio da aplicação do questionário Saint George's Respiratory Questionnaire (SGRQ). Metodologia: estudo transversal e analítico composto de 18 indivíduos com diagnóstico médico de DPOC internados em um hospital do norte do estado do Rio Grande do Sul, de ambos os sexos e com idade superior a 30 anos de idade. Foram utilizados dois questionários para coleta de dados, um específico de qualidade de vida em pacientes DPOC (SGRQ), e um questionário para caracterização da amostra. Resultados: a amostra foi composta de 18 indivíduos, sendo $9(50 \%)$ do sexo feminino. Quanto à faixa etária, 3 (16,7\%) tinham de 50-59 anos, 6 (33,33\%) de 60-69 anos e $9(50 \%)$ mais de 70 anos. Os valores obtidos nos escores do questionário SGRQ foram de 59,50 $\pm 16,86$ no domínio sintomas; $67,67 \pm 22,94$ no domínio atividade; $47,89 \pm 21,11$ no domínio impacto e o escore total de $54,44 \pm 16,97$. Conclusão: neste estudo a qualidade de vida dos indivíduos apresentou-se prejudicada e comprometida, mostrando, assim, a necessidade de direcionar atenção e cuidados a esse fator em pacientes com DPOC.

\section{Palavras-chave}

Doença pulmonar obstrutiva crônica; Qualidade de vida; Hospitalização.

\section{Fisißenectus}




\begin{abstract}
Introduction: Chronic Obstructive Pulmonary Disease (COPD) is characterized by airflow compromise in the bronchial tree, developing variable symptoms such as sputum, dyspnea and cough. Such symptoms of COPD compromise the functionality and social life of individuals, limiting their activities of daily living and thus affecting their quality of life. Objective: The present study had as objective to evaluate the quality of life in hospitalized individuals with medical diagnosis of COPD through the application of the Saint George's Respiratory Questionnaire (SGRQ) questionnaire. Methodology: This was a cross-sectional and analytical study of 18 individuals with a medical diagnosis of COPD hospitalized in a hospital in the north of the State of Rio Grande do Sul (RS), both men and women over 30 years of age. Two questionnaires were used for data collection, a specific quality of life in COPD patients (SGRQ), and a questionnaire to characterize the sample. Results: 18 individuals, 9 (50\%) were female. As for the age group, $3(16.7 \%)$ were $50-59$ years old, $6(33.33 \%)$ were 60-69 years old and 9 (50\%) were over 70 years old. The values obtained from the SGRQ domain scores were $59.50 \pm 16.86$ in the symptom domain; $67.67 \pm 22.94$ in the activity domain; $47.89 \pm 21.11$ in the impact domain and the Total score of $54.44 \pm 16.97$. Conclusion: In this study the quality of life of the individuals was impaired and compromised, thus showing the need to direct attention and care to the quality of life of patients with COPD.
\end{abstract}

\title{
Keywords
}

Chronic obstructive pulmonary disease; Quality of life; Hospitalization.

\section{Introdução}

$\infty \times \infty \times \infty \times \infty \times \infty \times \infty \times \infty \times \infty \times \infty \times \infty \times \infty \times \infty \times \infty)$

A doença pulmonar obstrutiva crônica (DPOC) é uma doença que tem como característica a limitação do fluxo aéreo e a inflamação das vias aéreas. A limitação do fluxo aéreo é na maioria das vezes progressiva, geralmente associada à resposta inflamatória pela inalação de substâncias tóxicas e gases nocivos que acabam comprometendo os pulmões ${ }^{1}$. Essa doença é típica da fase adulta, e geralmente acomete indivíduos com histórico de consumo de tabaco por longos períodos ${ }^{1-2}$. Vagarosamente a exposição aos agentes nocivos causam sintomas frequentes associados à doença como dispneia, tosse, produção de escarro, intolerância ao exercício, que se associam à depressão e à ansiedade ${ }^{1-3}$.

Os sintomas relacionados à DPOC e nos quadros de agudização ou exacerbação da doença, geralmente se associa a agravamento funcional, às vezes, irreversível, e, frequentemente, levam o indivíduo a procurar auxílio médico e internação hospitalar, como um método de auxílio para amenizar os sintomas, quando tardiamente pode elevar os custos da hospitalização ${ }^{4}$.
As alterações fisiológicas pulmonares decorrentes da doença geram impacto na qualidade de vida e no bem-estar dos indivíduos, e limitam a realização das atividades de vida diária ${ }^{3-5}$.

A descrição do conceito qualidade de vida não tem definição concreta. Um estudo realizado pela Organização Mundial da Saúde (OMS) que reuniu especialistas de várias partes do mundo para definir o conceito qualidade de vida, chegou a um consenso de que "[...] é a percepção do indivíduo sobre sua posição na vida, em relação à cultura que ele vive os valores que ele prega e em relações aos seus objetivos, expectativas, padrões e preocupações sobre a vida.", surgindo a necessidade da criação de instrumentos para quantificar dados subjeti$\operatorname{vos}^{6,8,9}$. Portanto, pode-se entender que o nível de qualidade de vida varia de acordo com as necessidades existentes de cada indivíduo ${ }^{7-9}$.

De certa forma nenhuma medida pode descrever precisamente quais são os impactos e a gravidade da DPOC na vida dos indivíduos, no entanto, atualmente, avaliar a qualidade de vida se tornou um componente essencial para medir o impacto da doença nos domínios físico e mental, sendo útil na elaboração e eficácia do tratamento ${ }^{1,6,10}$.

O termo "qualidade de vida" vem sendo citado com muita frequência e é decorrente das mudanças 
demográficas, sobretudo pela longevidade e pelo crescimento econômico que impõem a adoção de novos paradigmas nas políticas públicas e prática de saúde ${ }^{11}$.

O impacto da doença ocasiona limitações e transtornos físicos, que resultam em uma perda progressiva na capacidade para praticar atividades que eram realizadas rotineiramente. 0 que consequentemente leva o paciente a ter de enfrentar a diminuição da capacidade de agir com independência. A redução na capacidade de desempenhar atividades diárias resulta em alterações de equilíbrio emocional, afetivo e relacional, o que torna o indivíduo menos capaz de realizar as tarefas diárias e de se relacionar socialmente ${ }^{12-14}$.

Para auxiliar na melhora da compressão da qualidade de vida relacionada à saúde (QVRs) foram elaborados questionários para estimar as alterações de saúde aferidas do ponto de vista dos indivíduos. Esses questionários retratam uma tentativa de compreender o impacto da patologia na vida desses sujeitos ${ }^{8}$. 0 questionário mais aplicado para avaliar a qualidade de vida é o Medical Outcomes Study 36 - item Short Form Health Survey (SF-36). 0 SF-36 tem sua versão validada e traduzida para o português brasileiro. Entre outros questionários para avaliação da qualidade de vida, encontre-se o Saint George's Respiratory Questionnaire (SGRQ), questionário mais específico para avaliar a qualidade de vida nos pacientes com diagnósticos de DPOC, sendo sua validação e tradução para o português brasileiro realizada no ano de $2000^{8}$. Nesse contexto, o presente estudo teve como objetivo avaliar a qualidade de vida de indivíduos hospitalizados com DPOC por meio da aplicação do questionário SGRQ.

\section{Métodos}

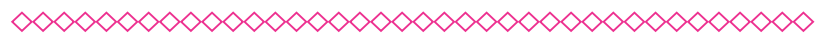

O delineamento do estudo foi transversal e analítico. Foram avaliados, neste estudo, 31 indivíduos, de ambos os sexos, internados no Hospital da Cidade no Município de Passo Fundo/RS, com diagnóstico médico de DPOC, durante o período de agosto a setembro de 2017.
A presente pesquisa foi aprovada pelo Comitê de Ética em pesquisa com seres humanos da Universidade de Passo Fundo (UPF), pela Resolução n. 466/2012, do Conselho Nacional de Saúde Parecer n. 2.186.945 e aprovação pelo Setor de Ensino e Pesquisa do Hospital da Cidade.

Os critérios de inclusão para o estudo foram: indivíduos com diagnóstico médico de DPOC, de ambos os sexos, com idade acima de 30 anos, capazes de compreender e assinar o termo de consentimento livre esclarecido (TCLE), que aceitaram participar do estudo. Como critérios de exclusão adotaram-se: indivíduos traqueostomizados, pacientes com sequelas neurológicas, oncológicos graves, e indivíduos com rebaixamento do nível do sensório ou cognição que impossibilitava a compreensão e preenchimento dos questionários.

Os indivíduos foram convidados a participar do estudo por meio de convite verbal individual, tendo livre arbítrio para aceitar ou recusa-lo. Todos foram esclarecidos sobre os procedimentos a serem realizados e os pacientes que aceitaram em participar do estudo assinaram o TCLE. Foram avaliados 31 pessoas com diagnóstico médico de DPOC internadas no período de agosto a setembro de 2017. Destes, depois da aplicação dos critérios de inclusão e exclusão da pesquisa, foram excluídos 13 por não se enquadrarem nos critérios do estudo. Sendo a amostra final do estudo composta de 18 indivíduos com DPOC. Esses indivíduos estavam internados por motivos diferentes, como pneumonia, edema pulmonar, tratamento cardiológico, pós-operatório de cirurgia pulmonar, cardíaca e intestinal. Todos os indivíduos passaram por uma entrevista individual realizada por dois entrevistadores para coleta de dados sociodemográficos para caracterização da amostra que compreendiam a idade e o sexo. Na sequência, foi aplicado o questionário de qualidade de vida SGRQ traduzido e validado para uso no Brasil.

O questionário SGRQ é específico para avaliar a qualidade de vida em portadores de DPOC e é composto de 50 itens divididos em três domínios: sintomas, atividade, e impacto da doença, cada item tem um valor. Esses valores são somados e calculados para cada domínio, o valor máximo do domínio sintomas é de 662,5, para o domínio atividade é 1209,1 e para o domínio impacto o valor é 
2117,8. 0 escore total é o resultado da soma calculada para cada domínio, que varia de zero a 100; os valores próximo de 100 significam pior qualidade de vida e próximos de zero significam melhor qualidade de vida.

Os dados foram codificados e digitados pelos pesquisadores em planilha do MS Excel. As variáveis categóricas foram descritas como frequência absoluta e relativa. As variáveis numéricas foram descritas como média \pm desvio padrão ou mediana, conforme apresentarem distribuição normal ou não normal.

\section{Resultados}

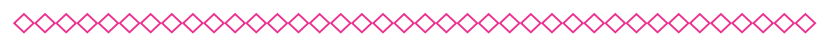

Foram avaliados 18 indivíduos que se encontravam internados no Hospital da Cidade com diagnóstico médico de DPOC. Na Tabela 1, está descrita a caracterização da população do estudo em relação ao sexo e à faixa etária.

0 resultado encontrado em relação à qualidade de vida dos indivíduos com DPOC avaliados por meio do questionário SGRQ foi apresentado na Tabela 2. Em todos os domínios os escores mantiveram-se elevados, acima de 50\%, no escore total médio, no domínio sintomas e principalmente no domínio atividade. No domínio impacto manteve-se levemente abaixo de $50 \%$

\section{Discussão}

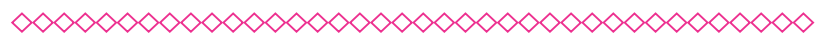

0 presente estudo avaliou 18 indivíduos com diagnóstico médico de DPOC, sendo 9 (50\%) indivíduos do sexo feminino. Indo de encontro ao estudo realizado por Barros et al. ${ }^{13}$, no qual os pesquisadores observaram predomínio do sexo masculino $(69,2 \%)$. Dados semelhantes foram encontrados no estudo de Carneiro et al. ${ }^{4}$ onde foram incluídos 45 doentes hospitalizados por DPOC agudizada no período em estudo, no qual foi encontrado predomínio do sexo masculino $(84,4 \%)$.

Neste estudo, observou-se o predomínio de indivíduos com faixa etária maior de 70 anos. Na literatura, foi verificado que os participantes de outros estudos tinham idade superior a 60 anos $^{6-13}$. E no estudo de Carneiro et al. ${ }^{4}$ também evidenciou Idade média de $68 \pm 12,4$ anos, caracterizando como indivíduos idosos. Verificou-se também que 0 nível de qualidade de vida de indivíduos com DPOC tende a agravar-se com o aumento da idade ${ }^{6}$.

Parte significativa dos artigos encontrados na literatura nacional e internacional que utilizaram o questionário SGRQ teve como objetivo avaliar e comparar o impacto na qualidade de vida durante o programa de reabilitação pulmonar, sendo um fator limitante para discussão dos dados encontrados neste estudo.

Observou-se que a qualidade de vida dos pacientes internados com diagnóstico de DPOC apresentou-se alterada, sendo que, os piores valores pontuados no SGRQ foram encontrados nos domínios sintomas $(59,50 \pm 16,86)$ e no domínio atividade $(67,67 \pm 22,94)$.

De acordo com o estudo de Carneiro ${ }^{4}$, para o qual foi aplicado o SGRQ em pacientes DPOC hospitalizados com depressão, observaram que 56\% dos doentes apresentava um estado depressivo. Valores mais elevados do SGRQ (isto é, indicativos de pior qualidade de vida) relacionaram-se com a presença de depressão $(R=0,69 ; p=0,02)$. 0 valor mediano da pontuação total do SGRQ foi de 50,6 pontos, corroborando com o presente estudo.

0 estudo de Dourado et al. ${ }^{12}$ desenvolvido na Faculdade de Medicina de Botucatu (São Paulo/SP) que utilizou o mesmo método de avaliação do presente estudo teve como objetivo avaliar a qualidade de vida, pelo questionário SGRQ, de 21 pessoas com DPOC que participavam de um programa de reabilitação pulmonar. Nesse estudo foram encontrados os seguintes escores para o domínio sintoma $52 \pm 17$, domínio atividade $54 \pm 24$, domínio impacto $43 \pm 17$ e no escore domínio total de $48 \pm 16$, mostrando valores inferiores quando comparados com os resultados do deste estudo.

Em outro estudo realizado no Brasil por Pereira et al. ${ }^{10}$, foram selecionados 42 pacientes com DPOC atendidos no Ambulatório de Pneumologia Hospital de Messejana, (Fortaleza/CE), com o objetivo de comparar a qualidade de vida com parâmetros funcionais e respiratórios, por meio do teste de caminhada de seis minutos e pelo questionário 
SGRQ. Todos os pacientes apresentaram alteração na qualidade de vida. Os valores demonstrados no SGRQ no domínio sintoma foi $50,28 \pm 25,60$; no domínio atividade de $63,45 \pm 24,51$ e no domínio impacto $49,77 \pm 25,08$; no escore total $54,0 \pm 22,7$. Os resultados encontrados nesse estudo no domínio atividade, no domínio impacto e no escore total foram semelhantes ao encontrado no presente estudo.

Na cidade Manuel Rocha Peixoto, na Unidade de Saúde familiar (USF), em Portugal, Barros et al. ${ }^{13}$ com o intuito de avaliar fatores que poderiam interferir na qualidade de vida, mobilizaram 65 pessoas diagnosticadas com DPOC a fizerem parte de uma avaliação dos parâmetros funcionais e respiratórios, seguida da aplicação do questionário SGRQ. Os pesquisadores observaram as seguintes médias: no domínio sintomas $53 \pm 19$,9; no domínio atividade $52 \pm 24,1$, no domínio impacto $36 \pm 24,1$ e a média do valor do escore total foi $44 \pm$ 20,9 . Os valores encontrados nesse o estudo foram menores em todos os domínios, principalmente no escore total, comparado com os resultados encontrado neste estudo (escore Total 54,44 $\pm 16,97$ ), 0 que reforça que a qualidade de vida dos pacientes DPOC no estudo atual encontra-se prejudicada.

\section{Conclusão}

$\infty \times \infty \times \infty \times \infty \times \infty \times \infty \times \infty \times \infty \times \infty \times \infty \times \infty \times \infty \times \infty)$

Avaliar a qualidade de vida nos indivíduos com DPOC se faz necessário para a melhor escolha na conduta e tratamento do paciente, para que os profissionais da saúde sintam-se mais seguros em suas práticas visando um melhor atendimento.

Diante dos resultados obtidos neste estudo, pode-se observar que é necessária atenção maior a esses pacientes, no sentido de promover um cuidado individualizado e especializado, melhorando a sua qualidade de vida.

A falta de estudo no âmbito hospitalar utilizando o questionário SGRQ para avaliação da qualidade de vida de indivíduos com diagnóstico médico de DPOC foi um limitante para a discussão mais detalhada. Nesse sentido, novos estudos mais delineados, com uma amostra maior e com a utilização do questionário no âmbito hospitalar, de modo a sensibilizar a comunidade científica sobre esse tema.

\section{Referências}

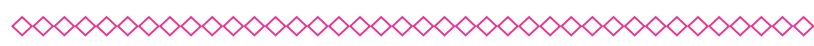

1. Buss AS, Silva LMCda. Estudo comparativo entre dois questionários de qualidade de vida em pacientes com DPOC. J Bras Pneumologia. 2009;35(4):318-24.

2. Croxton $T L$, Weinmann GG, Senior RM, Wise RA, Crapo JD, Buist AS. Clinical research in chronic obstructive pulmonary disease: needs and opportunities. Am J Respir Crit Care Med. 2003; 167:1142-49.

3. Camelier A, Rosa FW, Salim C, Nascimento OA, Cardoso F, Jardim JR. Using the Saint George's Respiratory Questionnaire to evaluate quality of life in patients with chronic obstructive pulmonary disease: validating a new version for use in Brazil. J Bras de Pneumologia. 2006;32(2):114-22.

4. Carneiro R, Sousa C, Pinto A, Almeida F, Oliveira $J R$, Rocha N. Risco de reinternamento na doença pulmonar obstrutiva crónica - Estudo prospectivo com ênfase no valor da avaliação da qualidade de vida e depressão. Rev Port de Pneumologia. 2010:16(5):759-77.

5. Erzinger GS, Mastroeni MF, Silva HE. Avaliação da qualidade de vida dos portadores de doença pulmonar obstrutiva crônica (DPOC) associada ao desempenho físico funcional. Rev Bras Ciência e Movimento. 2012;20(4):46-53.

6. Ståhl El, Lindberg A, Jansson SA, Rönmark E, Svensson $\mathrm{K}$, Andersson $\mathrm{F}$ et al. Health-related quality of life is related to COPD disease severity. Health and Quality Life Outcomes. 2005;3:56.

7. Britto RR, Santos CFF, Bueno FF. Reabilitação pulmonar e qualidade de vida dos pacientes portadores de DPOC. Rev Fisioterapia e Pesquisa. São Paulo. 2002;9(1):9-16.

8. Souza TC, Jardim JR, Jones P. Validação do Questionário do Hospital Saint George na Doença Respiratória (SGRQ) em pacientes portadores de 
doença pulmonar obstrutiva crônica no Brasil. J de Pneumologia. 2000;26(3)119-128.

9. WHOQOL GROUP. The development of the World Health Organization quality of life assessment instrument (the WHOQOL). In: Orley J, Kuyken W, editors. Quality of life assessment: international perspectives. Heigelberg: Springer Verlag;1994;41-60.

10. Pereira ED, Pinto R, Alcantara M, Medeiros $M$, Mota RMS. Influência dos parâmetros funcionais e respiratórios na qualidade de vida de pacientes com DPOC. J Bras de Pneumologia. 2009;35(8):730-6.

11. Carvalho ALdeO. Qualidade de vida após revascularização cirúrgica do miocárdio , angioplastia ou tratamento clínico : 10 anos de seguimento. [tese]. São Paulo: Universidade de São Paulo, 2013.

12. Dourado VZ, Antunes LCdeO, Carvalho LR, Godoy I. Influência de características gerais na qualidade de vida de pacientes com doença pulmonar obstrutiva crônica. J Bras de Pneumologia. 2004;30(3):207-14.

13. Barros M; Guimarães F, Sousa JCde. Fatores determinantes da qualidade de vida numa população de doentes com doença pulmonar obstrutiva crónica. Rev Port de Med Geral e Familiar. 2014;30:156-66.

14. Cedano S, Belasco AGS, Traldi F, Machado MCLO, Bettencourt ARdeC. Influence that sociodemographic variables, clinical characteristics, and level of dependence have on quality of life in COPD patients on long-term home oxygen therapy. J Bras de Pneumologia. 2012;38:331-8.

15. Farias GMdaS, Martins RML. Qualidade de Vida da Pessoa com Doença Pulmonar Obstrutiva Crónica. Millenium. 2013;45(20):195-209. 


\section{Anexos}

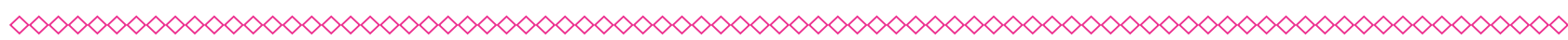

Tabela 1 - Caracterização da amostra

Tabela 1. Car acter ização da amostra. Passo Fundork - 2017.

\begin{tabular}{lll}
\hline Variáveis & $\mathrm{N}$ & W \\
\hline Sexo & & \\
\hline Masculino & 09 & 50,0 \\
Feminino & 09 & 50,0 \\
\hline Fàxas etárias & & \\
\hline 50-59 anos & 03 & 16,7 \\
60-69 anos & 06 & 33,3 \\
70 anos ou mais & $\infty$ & 50,0
\end{tabular}

Forte: elaborad'a peb autor. Legenda: $N^{\prime}=$ vabor absoluto; 米 vabor relativo. Variáveis descritas em toquência ahsoluta e reläi va.

Fonte: Elaborada pelos autores

(clique para voltar ao texto) 
Tabela 2 - Qualidade de vida de pacientes DPCO pelo SGRS

Tabela 2: Qualidade de vida de pacientes DPOC pelo SGRQ. Passo FundaRS - 2017.

\begin{tabular}{|c|c|}
\hline Domínio & Representação \\
\hline Sinto mas & $59,50 \pm 16,86$ \\
\hline Atividade & $67,67 \pm 22,94$ \\
\hline Impacto & $47,89 \pm 21,11$ \\
\hline Total & $54,44 \pm 16,97$ \\
\hline
\end{tabular}

Fonte: Elaborada pelos autores 